\title{
CrystEngComm
}

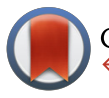

CrossMark \& click for updates

Cite this: CrystEngComm, 2016, 18 3422

Received 7th January 2016, Accepted 11th April 2016

DOI: $10.1039 / c 6 c e 00039 h$

www.rsc.org/crystengcomm

\section{Hydrophilic Pt nanoflowers: synthesis, crystallographic analysis and catalytic performance $\uparrow$}

\author{
Stefanos Mourdikoudis, ${ }^{a}$ Thomas Altantzis, ${ }^{\text {b }}$ Luis M. Liz-Marzán, ${ }^{\text {acd }}$ Sara Bals, ${ }^{\text {b }}$ \\ Isabel Pastoriza-Santos*a and Jorge Pérez-Juste*a
}

\begin{abstract}
Water-soluble Pt nanoflowers (NFs) were prepared by diethylene glycol-mediated reduction of Pt acetylacetonate $\left(\mathrm{Pt}(\mathrm{acac})_{2}\right)$ in the presence of polyethylenimine. Advanced electron microscopy analysis showed that the NFs consist of multiple branches with a truncated cubic morphology and different crystallographic orientations. We demonstrate that the nature of the solvent strongly influences the resulting morphology. The catalytic performance of the Pt NFs in 4-nitrophenol reduction was found to be superior to that of other nanoparticle-based catalysts. Additionally, the Pt NFs display good catalytic reusability with no loss of activity after five consecutive cycles.
\end{abstract}

\section{Introduction}

Platinum nanostructures are important in domains such as catalysis, sensors and fuel cells. ${ }^{1-5}$ In this context, a significant amount of work has been devoted to tailoring their morphology by properly tuning the experimental reaction conditions. $^{6-8}$ In this manner, shapes such as cubes, rods, dendrites, wires and polyhedra have been reported. In particular, the nanoflower configuration has attracted attention due to its surface roughness, excellent electrochemical performance and potential catalytic activity. Several synthetic routes toward Pt NFs have been reported in the literature, which usually yield hydrophobic Pt NFs. ${ }^{7,9}$ Sun et $a .^{10}$ reported a facile and large-scale synthesis of 100-200 nm 3D flower-like particles in aqueous medium, without using any capping agent, thus rendering them sensitive to aggregation. Other reports described the chemical synthesis of flower-like Pt nanoparticles in water, which were directly deposited on carbon nanotubes ${ }^{11}$ and the urea-assisted preparation of flower-like Pt arrays on other substrates. ${ }^{6}$ Monomorphic singlecrystalline platinum nanoflowers, soluble in ethanol, were obtained by Zhang and co-workers through an iodinemediated polyol process. ${ }^{8}$ Mohanty and colleagues presented

\footnotetext{
${ }^{a}$ Departamento de Quimica Física, Universidade de Vigo, 36310 Vigo, Spain

${ }^{b}$ EMAT, University of Antwerp, Groenenborgerlaan 171, B-2020 Antwerp, Belgium. E-mail: pastoriza@uvigo.es, juste@uvigo.es

${ }^{c}$ BioNanoPlasmonics Laboratory, CIC biomaGUNE, Paseo de Miramón 182, 20009 San Sebastián, Spain

${ }^{d}$ Ikerbasque, Basque Foundation for Science, 48013 Bilbao, Spain

$\dagger$ Electronic supplementary information (ESI) available: TEM and high resolution HAADF-STEM analysis, FTIR and XRD measurements, and a summary of the catalytic performance for the different nanoparticles. See DOI: 10.1039/c6ce00039h
}

an approach for one-pot, high-yield synthesis of hydrophilic nanoflowers of $\mathrm{Pt}$ as well as of other metals in the presence of an amino acid-based surfactant. ${ }^{12}$ Moreover, a sonoelectrodeposition method was developed by Heli and coworkers to produce Pt NFs hierarchically constructed from nanoparticles. ${ }^{13}$ We have recently reviewed the synthetic routes to obtain noble metal NPs (including Pt) with controlled composition, morphology, crystallinity and either a hydrophobic or hydrophilic surface. ${ }^{14}$

Hydrophilic Pt nanostructures are efficient catalysts for various reactions such as electrocatalytic oxidation of methanol, ${ }^{15}$ reduction of oxygen, ${ }^{11}$ and the Suzuki-Miyaura and Heck coupling reactions. ${ }^{16}$ Of much recent interest is the catalytic reduction of 4-nitrophenol (4-NP) to 4-aminophenol (4-AP), not only as a model reaction but also because nitrophenol is toxic in aqueous medium. One of the processes that have been proposed for nitrophenol degradation is the reduction of the nitro group to an amine, and spherical Pt nanoparticles, either hydrophilic ${ }^{17}$ or hydrophobic, supported onto a cellulose substrate $^{18}$ have been reported to catalyse this reaction.

In catalytic reactions, most substrates and products present poor water solubility. Although this is one of the major limitations of water in organic chemistry, it can be turned into an advantage, not just because water is not toxic, safe, inexpensive and can accelerate organic reactions, ${ }^{19}$ but it also facilitates separation, recycling and reuse of water-soluble catalysts. $^{20}$

We present here a one-pot method to prepare hydrophilic Pt nanoflowers, based on the solvothermal reduction of platinum acetylacetonate in diethylene glycol (DEG), in the presence of polyethylenimine (PEI). Since the reducing power of DEG depends on the reaction temperature, we first analyzed 
the effect of temperature on the size and shape of the obtained particles. We then analysed the importance of DEG in NF formation, through a comparison with $N, N$ dimethylformamide (DMF) as an alternative solvent and reducing agent. Anisole was also used for comparison, considering its role as a rather 'inert' solvent, with no reducing abilities. Apart from using conventional techniques such as X-ray diffraction and transmission electron microscopy, advanced electron tomography was also employed to obtain a 3D atomic scale reconstruction of the nanoparticles, so as to reveal their shape and crystallographic structure. We further analyzed the catalytic efficiency of the Pt nanoflowers in the hydrogenation of 4-nitrophenol (4-NP) to 4-aminophenol (4-AP) by sodium borohydride. ${ }^{21}$

\section{Experimental}

\section{Materials}

Branched PEI (Mw 25000 ), ethanol, anhydrous anisole (99.7\%), $\mathrm{NaBH}_{4}$ and 4-NP were provided by Sigma-Aldrich. $\operatorname{Pt}(\text { acac })_{2}(98 \%)$ was obtained from Strem Chemicals Inc., $\mathrm{NaOH}$ was from Merck and DEG was from Fisher Sci. (analytical grade, 99.98\%). All chemicals were used as received.

\section{Synthesis of Pt nanoflowers}

$30 \mathrm{~mL}$ of DEG containing $600 \mathrm{mM}$ PEI (the concentration refers to monomer units) were introduced into a three-neck flask. After sonication and magnetic stirring at room temperature for $20 \mathrm{~min}, 0.1 \mathrm{mmol}$ of $\mathrm{Pt}(\mathrm{acac})_{2}$ was added, forming a homogeneous solution with a yellowish colour. Then, the solution was rapidly heated to either $154^{\circ} \mathrm{C}$ or $240{ }^{\circ} \mathrm{C}$ (heating rate $\sim 15{ }^{\circ} \mathrm{C} \mathrm{min}^{-1}$ ). Within one hour at the selected temperature, the solution turned brown-black and it was kept at $154{ }^{\circ} \mathrm{C}$ or $240{ }^{\circ} \mathrm{C}$ for totally $24 \mathrm{~h}$ or $1 \mathrm{~h}$, respectively. Finally, it was allowed to cool down to room temperature. The Pt NFs were precipitated twice by centrifugation with excess ethanol $(8500 \mathrm{rpm}, 30$ min). Eventually, the nanoparticles were stored in $20 \mathrm{~mL}$ of Milli-Q water. The final Pt concentration was $3 \mathrm{mM}$ in the case of the synthesis at $154{ }^{\circ} \mathrm{C}$ and $4 \mathrm{mM}$ in the case of that at $240{ }^{\circ} \mathrm{C}$, due to some slight difference in the reaction yield.

\section{Characterisation}

Conventional TEM imaging was carried out using a JEOL JEM 1010 microscope operated at an acceleration voltage of $100 \mathrm{kV}$. The electron tomography series were acquired using a FEI Tecnai G2 electron microscope operated at $200 \mathrm{kV}$. A Fischione model 2020 single tilt tomography holder was used, and the series were acquired automatically with the use of Xplore3D software. All tilt series were acquired in HAADFSTEM mode with an angular range from $-68^{\circ}$ to $+76^{\circ}$ and a tilt increment of $2^{\circ}$. The alignment of the series was performed using the Inspect 3D software (FEI). The reconstruction of the series was performed by using the Simultaneous Iterative Reconstructive Technique (SIRT) as implemented in Inspect 3D. High resolution HAADF-STEM images were acquired using an aberration corrected cubed FEI Titan 50-80 electron microscope operated at $120 \mathrm{kV}$. The camera length was set to $115 \mathrm{~mm}$ in order to guarantee an incoherent imaging mode. Prior to using the HAADF-STEM images as inputs for the tomographic reconstruction, a constant background value was subtracted. The high resolution reconstruction was obtained by minimizing the L1 norm of the object simultaneously with the projection distance, as explained in ref. 24. The colloidal solutions were dried overnight under dynamic vacuum to prepare solid powder samples suitable for X-ray diffraction (XRD) measurements. XRD was measured using a Siemens D-5000 diffractometer with $\mathrm{Cu} \mathrm{K} \alpha$ radiation (1.54059 $\AA$ ). $Z$-potential values were acquired via electrophoretic mobility measurements by taking the average of five measurements at the stationary level, using a Zetasizer Nano S (Malvern Instruments) with a He-Ne laser operating at $633 \mathrm{~nm}$ and a detection angle of $173^{\circ}(4 \mathrm{~mW})$. ICP-OES elemental analyses were performed after digestion of the samples with aqua regia. FTIR spectra were recorded using a Nicolet 6700 FTIR spectrometer with a resolution of 4 $\mathrm{cm}^{-1}$ using previously dried powder of platinum nanoflowers in the form of $\mathrm{KBr}$-based pellets.

\section{4-Nitrophenol to 4-aminophenol reduction}

In a $1 \mathrm{~cm}$ quartz cuvette, $1.34 \mathrm{~mL}$ of Milli-Q water was added, followed by the addition of $400 \mu \mathrm{L}$ of an aqueous solution containing $0.1 \mathrm{mg}$ of Pt NFs $(21 \mathrm{~nm})$. Then, $750 \mu \mathrm{L}$ of a freshly prepared $0.1 \mathrm{M} \mathrm{NaBH}_{4}$ solution (9.1 mM in $\mathrm{NaOH}$ ) was added, followed by $13 \mu \mathrm{L}$ of $9.61 \mathrm{mM}$ 4-nitrophenol aqueous solution. A diode-array UV-visible spectrophotometer (Agilent 8453) was employed to monitor the reaction by recording absorbance spectra every $30 \mathrm{~s}$, at room temperature. The 4-aminophenol product was identified by monitoring the changes with time of the absorbance at $400 \mathrm{~nm}$, where the maximum variations take place. The apparent observed rate constants $k_{\text {app }}$ were calculated from the plots of absorbance $v s$. time, using the first-order rate equation:

$$
A_{t}=A_{\mathrm{f}}+\Delta A \mathrm{e}^{-k t}
$$

where $A_{t}$ is the absorbance of the reaction mixture at time $t$, $A_{\mathrm{f}}$ is the final absorbance and $k$ is the apparent observed first-order rate constant of the reaction.

\section{Recyclability test}

When the catalytic reduction of 4-NP to 4-AP was deemed completed, $13 \mu \mathrm{L}$ of $4-\mathrm{NP}$ was added again to the reaction solution, and the kinetic studies were continued after mixing. This procedure was repeated several times.

\section{Results and discussion}

Uniform platinum nanoflowers (Fig. 1 and S1 $\dagger$ ) were obtained by the reduction of $\mathrm{Pt}(\mathrm{acac})_{2}$ in a PEI-containing DEG solution at high temperature (see Experimental). DEG 

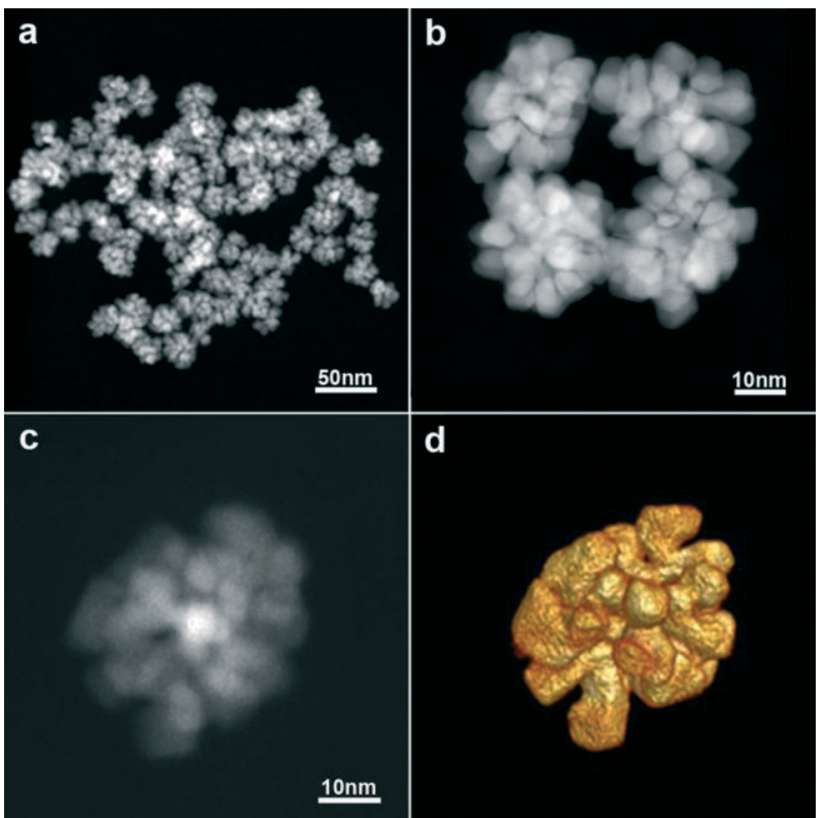

Fig. 1 a-c) HAADF-STEM images of the Pt nanoflowers and d) a 3D representation of the reconstructed volume of the nanoflower shown in c.

is expected to act as both a solvent and a reducing agent, whilst PEI is a capping agent providing high colloidal stability in water $(\zeta \sim+40 \mathrm{mV})$, even for several months. The presence of PEI on the particle surface was further characterized by FTIR spectroscopy. As shown in Fig. S2, $\dagger$ the spectrum displayed peaks at 2925 and $2852 \mathrm{~cm}^{-1}$, assigned to the asymmetric and symmetric vibrations of the $\mathrm{CH}_{2}$ group, respectively, and at $1450 \mathrm{~cm}^{-1}$, attributed to the in-plane bending of the $\mathrm{CH}_{2}$ in PEI. Besides, the peaks corresponding to the bending vibration of the $\mathrm{NH}$ group $\left(1635 \mathrm{~cm}^{-1}\right)$ and the stretching vibration of the $\mathrm{C}-\mathrm{N}$ groups $\left(1160 \mathrm{~cm}^{-1}\right)$ of PEI were clearly distinguished. ${ }^{22}$ In addition, the high boiling point of DEG (244-245 ${ }^{\circ} \mathrm{C}$ ) allows us to use it within a wide temperature range, thus modifying its reducing capability; a higher reaction temperature allows sufficient rearrangement of atoms during growth, resulting in highly crystalline materials. ${ }^{23}$ The reaction was thus performed at 154 and $240{ }^{\circ} \mathrm{C}$ (Fig. 1 and S1 $\dagger$ ), showing that the particle morphology and uniformity were not affected, but particle size was slightly modified (21 nm vs. $34 \mathrm{~nm}$, on average, for $154^{\circ} \mathrm{C}$ and $240{ }^{\circ} \mathrm{C}$, respectively), and the reaction kinetics were markedly different ( $24 \mathrm{~h} v s .1 \mathrm{~h}$, respectively).

Analysis by high angle annular dark-field scanning transmission electron microscopy (HAADF-STEM) showed that the NFs consist of branches and leaves with different crystallographic orientations. Since STEM images only provide 2D projections, electron tomography was used to obtain reliable 3D characterization of the morphology of the Pt NFs. As shown in Fig. 1c and d, the 3D reconstruction of a representative Pt NF shows that the branches are not thin leaves but rather exhibit a faceted morphology. XRD characterization additionally reveals that the NFs comprise high purity face- centred cubic (fcc) crystals (Fig. S3†). The XRD peaks matched well with Pt JCPDS card no.: 00-004-0802 and can be assigned to the crystal planes (111), (200), (220), and (311). Application of Scherrer's equation for the (111) peak at $39.8^{\circ}$ yielded a crystalline monodomain size of around $7 \mathrm{~nm}$, which is in agreement with the values obtained by TEM for the size of a single "petal". Taking into account that TEM analysis gives an average particle size of 21 or $34 \mathrm{~nm}$ (for 154 and $240{ }^{\circ} \mathrm{C}$, respectively), we expect that the NFs comprise multiple crystallographic domains, which was confirmed by high-resolution STEM. Fig. S4† clearly shows that the petals display different crystallographic orientations with twin boundaries between them. Aiming to obtain a better understanding of the morphology of the individual petals comprising the whole $\mathrm{NF}$, a $3 \mathrm{D}$ reconstruction at the atomic scale was obtained (Fig. 2 and $\mathrm{S} 5 \dagger$ ). Three high resolution images from a tip of a petal were acquired along different $<110>$ zone axes (Fig. 2a-c) and then used as input for a compressive sensing-based reconstruction algorithm. ${ }^{24}$ As can be observed in Fig. 2, the morphology of the investigated petal corresponds to a truncated cube. A model of such a cube, generated using VESTA (a 3D visualization program for electronic and structural analysis), ${ }^{25}$ is presented in Fig. 2d, which is the most dominant morphology in the branches. This comprehensive morphological and crystallographic investigation clearly demonstrated the 'faceted' configuration of the petals, which are thus the 'constituting units' for the NFs.

Our results are different from those presented by Yin et al., ${ }^{8}$ where the use of iodine ions resulted in the formation of monomorphic single-crystalline Pt nanoflowers. In their case, the continuous fringes from the core to the petals suggest that the latter grow epitaxially, and all the Pt atoms are arranged in one periodicity, providing a uniform orientation for all the branches or petals. ${ }^{8,12}$ The polycrystalline nature of our nanoflowers indicates a distinct formation mechanism. It is likely that the individual nanocrystals coalesce at the early stages due to competition between the remaining Pt precursors and PEI (in constant attachment/detachment induced by the high temperature). ${ }^{7}$ Such a 'coalescence' mechanism may be due to a slow reduction rate, resulting in a large amount of monomers that crystallize into a large number of nuclei, which are near each other in an environment rich in Pt monomers. This situation facilitates a 'coalescence' growth mode, leading to progressive condensation of the nanocrystals into the nanoflower structures. $^{7}$ The presence of PEI helps controlling the extent of such coalescence, hindering the formation of large platinum aggregates.

The importance of using DEG as a solvent was confirmed by carrying out the reaction in either DMF or anisole, keeping all the other conditions unchanged. Nanoparticles with a dendrite shape were obtained with DMF (in agreement with ref. 26, Fig. S6a $\dagger$ ), whereas oligopods were the main morphology when anisole was used (Fig. S6b $\dagger$ ). The differences in oxidation potential and/or coordination behaviour in various 

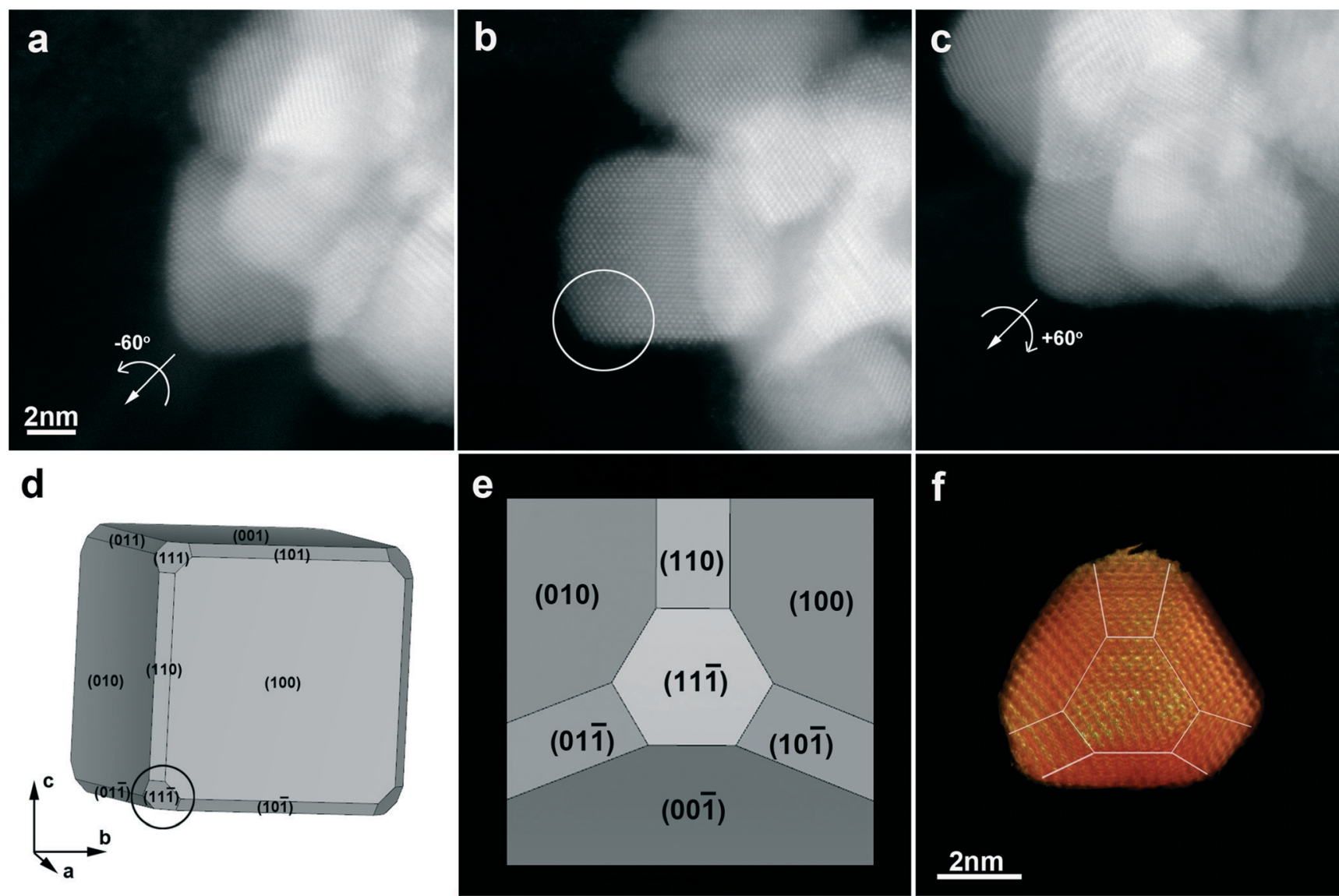

Fig. 2 a-c) High resolution HAADF-STEM images of the branch used for the atomic scale reconstruction, oriented along three different $<110>$ zone axes. The reconstructed part of the branch is the lower left corner, indicated by the white circle in b. d) A model of a truncated cube made using VESTA, which is the most dominant morphology in the branches. The cube is on the same orientation as the branch in Fig. 2b. e) The model along the [11-1] orientation, in order to be compared with the reconstructed volume, which is also oriented in the same direction. f) 3D representation of the reconstructed volume of the corner of the branch, where the indexing of the facets is also included. The facets are mainly $\{110\}$ and $\{100\}$, and for the acquisition of the high resolution series, the branch was tilted around the corner of the cube, the [11-1] axis. In a, the crystal is tilted $-60^{\circ}$ with respect to $\mathrm{b}$, while in $\mathrm{c}$, it is tilted $+60^{\circ}$.

solvents have been reported to result in varying sizes and shapes. ${ }^{27-30}$

Finally, the catalytic performance of $21 \mathrm{~nm}$ platinum nanoflowers was tested for the conversion of 4-nitrophenol (4-NP) to 4-aminophenol (4-AP) by sodium borohydride (see the inset in Fig. 3a). 4-Aminophenol is an important intermediate in the preparation of drugs, lubricants, and dyes. ${ }^{3,31}$ Therefore, it is desirable to develop efficient and reusable catalytic systems for the preparation of 4-AP. This reaction can be easily monitored by UV-visible spectroscopy, since nitrophenolate ions and 4-AP display absorption bands centred at $400 \mathrm{~nm}$ and $300 \mathrm{~nm}$, respectively (Fig. 3a). Although sodium borohydride is regarded as a strong reducing agent, it can be considered inert for 4-nitrophenol reduction in the absence of a catalyst. The presence of the Pt NFs in the reaction medium leads to a gradual decrease of the band for nitrophenolate ions, along with a gradual increase in intensity of the 4-AP band, which indicates the catalytic conversion of 4-NP to 4-AP (Fig. 3a). It should be pointed out that a large excess of $\mathrm{NaBH}_{4}$ with respect to 4-NP allows us to treat the ki- netics of the catalytic process as a pseudo-first order reaction. The apparent rate constant $\left(k_{\text {app }}\right)$ was assumed to be proportional to the surface $S$ of the metal and also to the sodium borohydride concentration, therefore, the kinetic constant ( $\left.k_{\text {app }}\right)$ can be defined as

$$
-\frac{\mathrm{d}[4 \mathrm{NP}]}{\mathrm{d} t}=k_{\text {app }}[4 \mathrm{NP}]=k_{\mathrm{obs}} S\left[\mathrm{NaBH}_{4}\right][4 \mathrm{NP}]
$$

where $k_{\text {obs }}$ is the observed pseudo-first order rate constant, $S$ is the metal catalyst surface, $[4-\mathrm{NP}]$ is the concentration of 4-NP at a given time $t$ and $\left[\mathrm{NaBH}_{4}\right]$ is the sodium borohydride concentration (assumed to be constant).

Fig. 3b shows the reaction kinetics through a plot of the absorbance at $400 \mathrm{~nm} v s$. reaction time, as well as a fit to a first-order rate equation (see the experimental section), which yields an observed rate constant of $7.0 \times 10^{-4} \mathrm{~s}^{-1}$. Additionally, the linearized data for first-order analysis also highlight the high-quality first-order nature of the reaction. 

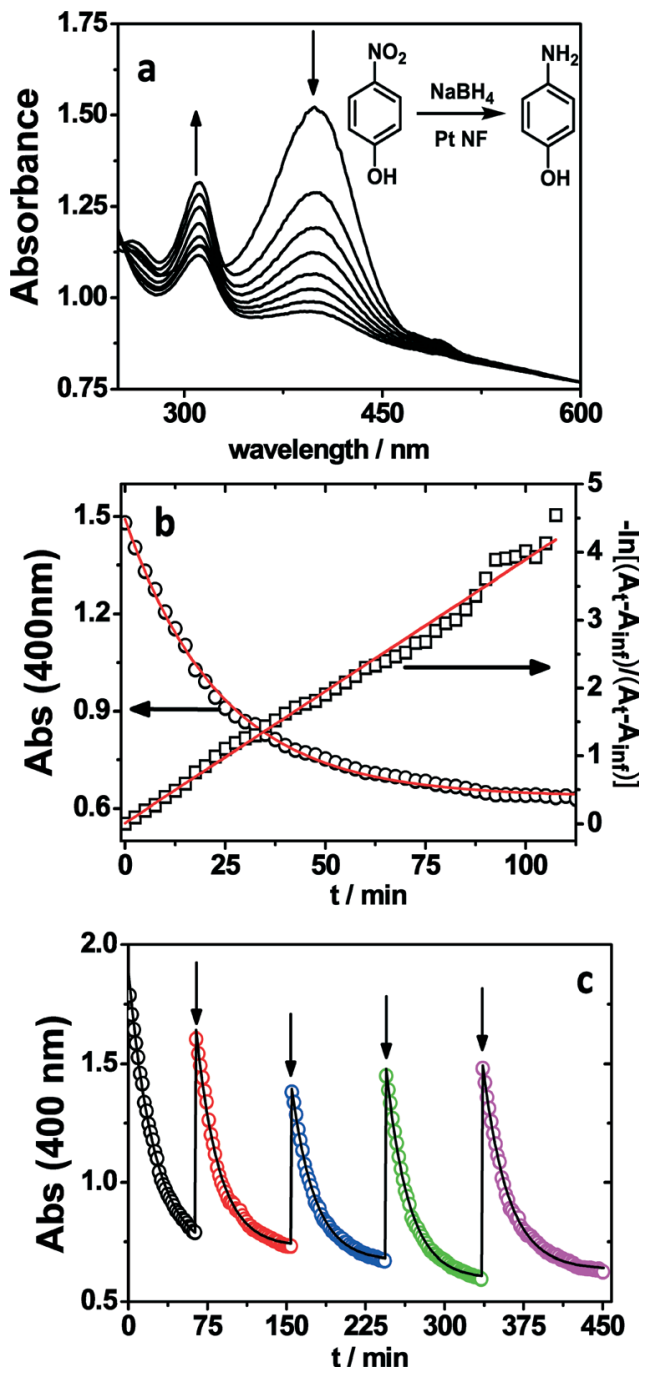

Fig. 3 a) Spectral evolution of a mixture of 4-NP and Pt nanoflowers upon borohydride addition. [4-NP] $=0.05 \mathrm{mM}, 0.1 \mathrm{mg}$ of Pt NF and $\left[\mathrm{NaBH}_{4}\right]=0.03 \mathrm{M}, T=25^{\circ} \mathrm{C}$. b) Kinetic trace of the absorbance at $400 \mathrm{~nm}$ during the reduction of 4-NP, and linearized data for firstorder analysis corresponding to Fig. 3a. c) Absorbance kinetic traces at $400 \mathrm{~nm}$, registered during the sequential reduction of 4-NP. The arrows indicate the times at which 4-NP was added to obtain [4-NP] = $0.05 \mathrm{mM}$. The line represents the best fit to a first-order rate equation.

In order to compare our results with previous reports, the normalized rate constant $\left(k_{\text {nor }}\right)$ was determined by

$$
k_{\text {nor }}=k_{\text {app }} /\left(m\left[\mathrm{NaBH}_{4}\right]\right)
$$

normalizing $k_{\text {app }}$ with respect to the total amount of the catalyst $(m)$ and the borohydride concentration $\left(\left[\mathrm{NaBH}_{4}\right]\right)$. The metallic Pt content was determined by ICP-OES spectroscopy to be $0.1 \mathrm{mg}$. Thus, the $k_{\text {nor }}$ value obtained for the Pt NFs (21 $\mathrm{nm}$ ) was $c a .233 .3 \mathrm{~g}^{-1} \mathrm{~s}^{-1} \mathrm{M}^{-1}$. This value is greater than the $k_{\text {nor }}$ estimated for other nanoparticle-based catalysts, such as Pt black (69 $\left.\mathrm{g}^{-1} \mathrm{~s}^{-1} \mathrm{M}^{-1}\right){ }^{32}$ Au@citrate $\left(27.6 \mathrm{~g}^{-1} \mathrm{~s}^{-1} \mathrm{M}^{-1}\right)^{32}$ or $\mathrm{Ag}\left(68.9 \mathrm{~g}^{-1} \mathrm{~s}^{-1} \mathrm{M}^{-1}\right)^{33}$ and $\mathrm{Au}\left(77.5 \mathrm{~g}^{-1} \mathrm{~s}^{-1} \mathrm{M}^{-1}\right)^{34}$ dendrites (see Table $\mathrm{S} 1 \dagger$ ), indicating their superior performance.
To check the reproducibility/reusability of the Pt NFs as catalysts, the nitro to amine conversion was repeated several times, using the same colloidal dispersion, through the sequential addition of 4-NP to an aqueous solution containing excess borohydride and a constant concentration of the platinum nanoflowers. Fig 3c shows the kinetic trace at $400 \mathrm{~nm}$ as well as the fit to a pseudo first-order reaction for each 4-NP addition. It is obvious from this plot that the first-order kinetic behaviour was perfectly reproducible, as predicted by eqn (1). The catalyst reusability with a yield of 100\% 4-AP after the fifth cycle indicated no loss in catalytic activity. Moreover, the consistency of the calculated apparent rate constant, $k_{\text {app}}$, alongside the different additions/cycles ( 5 in total) of 4 -NP $\left(7.0 \times 10^{-4} \mathrm{~s}^{-1}, 7.8 \times 10^{-4} \mathrm{~s}^{-1}, 7.7 \times 10^{-4} \mathrm{~s}^{-1}, 7.8 \times 10^{-4}\right.$ $\mathrm{s}^{-1}$, and $7.0 \times 10^{-4} \mathrm{~s}^{-1}$ ) is a clear evidence for the remarkable stability of the Pt NFs in the reaction medium, as well as the reproducibility of their catalytic activity. Typically, the deactivation of catalytic efficiency during recycling, when dealing with colloidal catalysts, has been ascribed to the agglomeration of the metal nanoparticles leading to a decrease in the active surface area. ${ }^{34}$ In our case, the high catalytic reusability can be ascribed to the high stability provided by the positively charged polymer PEI, even at high ionic strength. The Pt nanoparticles were observed by TEM after the catalytic cycles and, as shown in Fig. S7, $\dagger$ the reduction of 4-NP did not affect the nanoflower morphology.

\section{Conclusions}

To conclude, uniform platinum nanoflowers were synthesized via a one-pot protocol based on the solvothermal reduction of Pt(acac $)_{2}$ in DEG, employing PEI as a capping agent. The presence of PEI on the particle surface provides them high stability in water. Complete structural characterization via HAADF-STEM and 3D reconstruction by electron tomography showed that the NFs consisted of multiple fcc branches (around $7 \mathrm{~nm}$ ) with a truncated cubic morphology and different crystallographic orientations. Analysis of the catalytic reduction of 4-nitrophenol to 4-aminophenol with sodium borohydride showed superior catalytic activity of the Pt NFs compared to other metal nanoparticles, as well as good reusability.

\section{Acknowledgements}

The authors would like to thank J. Millos for the XRD experiment and R. Lomba for the ICP-OES elemental analysis measurements at the CACTI Institute in Vigo. S. Rodal-Cedeira is acknowledged for the FTIR measurement. This research project was implemented within the framework of the Action "Supporting Postdoctoral Researchers" of the Operational Program "Education and Lifelong Learning" (Action's Beneficiary: General Secretariat for Research and Technology of Greece) and was co-financed by the European Social Fund (ESF) and the Greek State [project code PE4(1546)]. This work has been also supported by the Spanish MINECO (grants MAT2013- 
45168-R and MAT2013-46101-R) and by the Xunta de Galicia/ FEDER (Grant No. GPC2013-006; INBIOMED/FEDER "Unha maneira de facer Europa"). S. B. acknowledges funding from the European Research Council under the Seventh Framework Program (FP7) (ERC Grant No. 335078 COLOURATOMS).

\section{Notes and references}

1 T.-L. Hsieh, H.-W. Chen, C.-W. Kung, C.-C. Wang, R. Vittal and K.-C. Ho, J. Mater. Chem., 2012, 22, 5550.

2 L. Wei, Y. J. Fan, H. H. Wang, N. Tian, Z. Y. Zhou and S. G. Sun, Electrochim. Acta, 2012, 76, 468.

3 Z. D. Pozun, S. E. Rodenbusch, E. Keller, K. Tran, W. Tang, K. J. Stevenson and G. Henkelman, J. Phys. Chem. C, 2013, 117, 7598.

4 D. R. Rolison, Science, 2003, 299, 1698.

5 D. Zhai, B. Liu, Y. Shi, L. Pan, Y. Wang, W. Li, R. Zhang and G. Yu, ACS Nano, 2013, 7, 3540.

6 M. Zhang, J.-J. Lv, F.-F. Li, N. Bao, A.-J. Wang, J.-J. Feng and D.-L. Zhou, Electrochim. Acta, 2014, 123, 227.

7 S. I. Lim, I. Ojea-Jiménez, M. Varon, E. Casals, J. Arbiol and V. Puntes, Nano Lett., 2010, 10, 964.

8 J. Yin, J. Wang, M. Li, C. Jin and T. Zhang, Chem. Mater., 2012, 24, 2645.

9 S. Maksimuk, X. Teng and H. Yang, J. Phys. Chem. C, 2007, 111, 14312.

10 S. Sun, D. Yang, D. Villers, G. Zhang, E. Sacher and J.-P. Dodelet, Adv. Mater., 2008, 20, 571.

11 S. Ghosh and C. R. Raj, J. Phys. Chem. C, 2010, 114, 10843.

12 A. Mohanty, N. Garg and R. Jin, Angew. Chem., Int. Ed., 2010, 49, 4962.

13 H. Heli, N. Sattarahmady, R. Dehdari Vais and A. R. Mehdizadeh, Sens. Actuators, B, 2014, 192, 310.

14 L. Polavarapu, S. Mourdikoudis, I. Pastoriza-Santos and J. Perez-Juste, CrystEngComm, 2015, 17, 3727.

15 Z. H. Lin, M. H. Lin and H. T. Chang, Chem. - Eur. J., 2009, 15, 4656.

16 K. Zhou and Y. Li, Angew. Chem., Int. Ed., 2012, 51, 602.
17 S. M. El-Sheikh, A. A. Ismail and J. F. Al-Sharab, New J. Chem., 2013, 37, 2399.

18 G. Zheng, K. Kaefer, S. Mourdikoudis, L. Polavarapu, B. Vaz, S. Cartmell, A. Bouleghlimat, N. J. Buurma, L. Yate, A. R. de Lera, L. M. Liz-Marzán, I. Pastoriza-Santos and J. Pérez-Juste, J. Phys. Chem. Lett., 2015, 6, 230.

19 A. Manna and A. Kumar, J. Phys. Chem. A, 2013, 117, 2446.

20 Handbook of Asymmetric Heterogeneous Catalysis, ed. K. Ding and Y. Uozumi, WILEY-VCH Verlag GmbH \& Co. KGaA, Weinheim, 2008.

21 S. Gu, S. Wunder, Y. Lu, M. Ballauff, R. Fenger, K. Rademann, B. Jaquet and A. Zaccone, J. Phys. Chem. C, 2014, 118, 18618.

22 T. Yang, A. Hussain, S. Bai, I. A. Khalil, H. Harashima and F. Ahsan, J. Controlled Release, 2006, 115, 289.

23 K. Tao, S. Song, J. Ding, H. Dou and K. Sun, Colloid Polym. Sci., 2011, 289, 361.

24 B. Goris, S. Bals, W. van den Broek, E. Carbó-Argibay, S. Gómez-Graña, L. M. Liz-Marzán and G. van Tendeloo, Nat. Mater., 2012, 11, 930.

25 K. Momma and F. Izumi, J. Appl. Crystallogr., 2011, 44, 1272.

26 S. Mourdikoudis, M. Chirea, T. Altantzis, I. Pastoriza-Santos, J. Perez-Juste, F. Silva, S. Bals and L. M. Liz-Marzán, Nanoscale, 2013, 5, 4776.

27 A. J. Biacchi and R. E. Schaak, ACS Nano, 2011, 5, 8089.

28 R. Choi, S. I. Choi, C. H. Choi, K. M. Nam, S. I. Woo, J. T. Park and S. W. Han, Chem. - Eur. J., 2013, 19, 8190.

29 S. Mourdikoudis, V. Colliere, P. Fau and M. L. Kahn, Dalton Trans., 2014, 43, 8469.

30 F. J. Douglas, D. A. MacLaren and M. Murrie, RSC Adv., 2012, 2, 8027.

31 M. J. Vaidya, S. M. Kulkarni and R. V. Chaudhari, Org. Process Res. Dev., 2003, 7, 202.

32 J.-J. Lv, A.-J. Wun, X. Ma, R.-Y. Xiang, J.-R. Chen and J.-J. Feng, J. Mater. Chem. A, 2015, 3, 290.

33 W. Ye, Y. Chen, F. Zhou, C. Wang and Y. Li, J. Mater. Chem. C, 2012, 22, 18327.

34 Y. Qiu, Z. Ma and P. Hu, J. Mater. Chem. A, 2014, 2, 13471. 